\title{
Simposio \\ ENFERMEDAD POR TAENIA SOLIUM: \\ TÓPICOS SELECTOS
}

\section{Estrategias moleculares para el serodiagnóstico de la teniasis/cisticercosis}

\author{
Gisela García \\ Grupo de Parasitología, Universidad de Antioquia, Medellín, Colombia
}

Las enfermedades causadas por Taenia solium en el humano, a la fecha, se encuentran categorizadas dentro de las "enfermedades desatendidas" y son consideradas por la Organización Mundial de la Salud (OMS) y el International Task Force for Disease Eradication (ITFDE), como un problema de salud humana y porcina potencialmente erradicable, que exige la implementación de procedimientos de diagnóstico sensibles, específicos y confiables, los cuales, a pesar de los esfuerzos de la comunidad científica, aún son insuficientes.

Sin embargo, y aunque las cifras son poco confiables, la OMS estima que en el mundo, aproximadamente, 50 millones personas están infectadas con T. solium y que cerca de 50.000 mueren anualmente por cisticercosis. En Colombia, las enfermedades causadas por $T$. solium, son prevalentes y la manifestación neurológica es la enfermedad parasitaria humana del sistema nervioso central más importante.

El diagnóstico definitivo de estas enfermedades está basado en técnicas que permiten la detección directa del parásito, las cuales se desempeñan eficientemente en el diagnóstico de la teniasis, pero que son en muchos casos inespecíficas, para la detección de cisticercos en tejidos, además de costosas y poco accesibles. En este sentido y sabiendo que en la cisticercosis se induce una respuesta inmunitaria humoral mediada por anticuerpos, los resultados seropositivos se han convertido en un marcador diagnóstico importante y la OMS establece entre sus criterios diagnósticos, una serológica positiva, la cual, en combinación con otros criterios, permite un diagnóstico definitivo de cisticercosis.

Muchas de las técnicas serológicas empleadas a la fecha para el diagnóstico de esta parasitosis, usan como fuente de antígeno, mezclas proteicas (antígenos crudos somáticos totales, semipurificados o glucoproteínas), cuya obtención requiere de la disposición de diferentes estadios parasitarios (huevos, larvas o adultos) y, por tanto, de diferentes modelos animales y procedimientos laboriosos; todas estas características conducen a datos variables y discordantes de sensibilidad, especificidad y reproducibilidad, en las técnicas que utilizan este tipo de antígenos.

Como una alternativa razonable para superar estos obstáculos, desde hace algunas décadas se inició la manufactura de proteínas heterólogas, usando técnicas de ingeniería genética, las cuales proveen los medios necesarios para obtener calidad y cantidad de antígenos, de los cuales se conozcan sus características moleculares y bioquímicas, es decir, antígenos de "segunda generación".

El desarrollo de las tecnologías del ADN recombinante ha mejorado la efectividad de las pruebas utilizadas para la detección de anticuerpos, al prescindir del organismo natural como fuente de proteínas y permitir estudiar de forma individual la potencialidad de un antígeno para el serodiagnóstico. Estas técnicas también hacen posible contar con las secuencias de ADNA que codifican para las proteínas y la secuencia de sus aminoácidos, abriendo la puerta a un estudio más profundo de estas macromoléculas.

Así, pues, el uso de herramientas genéticas, bioquímicas y moleculares para la obtención de proteínas recombinantes, como antígenos de segunda generación, se ha convertido en el top de las pruebas serológicas, ya que se pueden obtener antígenos definidos, de forma reproducible, controlada, con alta pureza y a bajo costo.

Para la selección de los antígenos que se desean obtener recombinantes, se han implementado varias estrategias, como la homología antigénica entre diferentes especies de Taenia, la reacción previamente observada o reportada con antígenos somáticos o de primera generación de $T$. solium, y de los resultados obtenidos con los extractos glucoproteícos del fluido vesicular de $T$. solium, cuyo uso en un inmunoblot al momento, esta 
validado por la OMS para el diagnóstico de la cisticercosis humana. Como una estrategia moderna, algunos autores vienen trabajando con secuencias de ADN reportadas en bases de datos públicas, que codifican para proteínas promisorias, pero que necesitan ser validadas y mejoradas, por ejemplo, mediante la construcción de proteínas quiméricas, la detección de péptidos antigénicos y su obtención sintética o recombinante, entre otras.

Las principales proteínas que se han evaluado para el serodiagnóstico de la cisticercosis son: NC3-NC9 con masa molecular de $8 \mathrm{kDa}$ y $13 \mathrm{kDa}$, respectivamente; CyDa-rTsM10 (subunidad de 10 $\mathrm{kDa}$ del complejo proteico de $150 \mathrm{kDa}$, del fluido vesicular de $T$. solium), la cual solo reacciona con anticuerpos de pacientes con neurocisticercosis activa. La familia de proteínas de $8 \mathrm{kDa}$, a la cual pertenecen, aproximadamente, 21 glucoproteínas de bajo masa molecular, que tienen secuencias señales de clivaje, después del cual, resultan proteínas maduras de 66-67 aminoácidos, con una masa molecular de $8 \mathrm{kDa}$, aproximadamente.

Tanto NC3-NC9 como CyDa-rTsM10 y la familia de proteínas de $8 \mathrm{kDa}$, han sido obtenidas fusionadas a la glutatión-S-transferasa, y su origen ha sido de librerías de CADN de cisticercos de T. solium. Para estas proteínas se reportan sensibilidades que van del 33 al $96 \%$ y especificidades del 91 al $96 \%$. Ag1V1 y Ag2, dos proteínas miembro de la familia de proteínas de $8 \mathrm{kD}$, se obtuvieron en una quimera conocida como RecTs, cuyo uso mostró datos de sensibilidad del $89,7 \%$ y especificidad del $100 \%$. De esta familia también se reporta la construcción y evaluación de péptidos sintéticos (Ts14, TsRs1, TsR2 var1, Ts18, Ts18 var1, Ts18 var3, Ts18 var4, Ts18 var6, Ts18 var8) que muestran datos muy variables de sensibilidad y especificidad.

Otra proteína reportada es la rGP50, uno de los componente antígenos glucoproteicos validados por la OMS, la cual fue expresada en báculovirus para tratar de conservar su antigenicidad, obteniéndose sensibilidad de $94,7 \%$ y especificidad de $93,8 \%$ $y$, finalmente, se reportan a KETc1, KETc12 cuyo origen es una librería de cDNA de $T$. crassiceps, las cuales mostraron una sensibilidad del 40 y 87,5 $\%$, respectivamente y $100 \%$ de especificidad.

Teniendo en cuenta estos resultados, se observa que el uso de antígenos recombinantes ha logrado mejorar la especificidad que se obtiene con los antígenos crudos usados convencionalmente. Sin embargo, se reporta variabilidad en sensibilidad, especificidad y reproducibilidad, cuando estos antígenos son usados, por ejemplo, para el diagnóstico de infecciones con cargas parasitarias leves, moderadas o altas, cuando el antígeno pierde su parte glúcida, cuando se utilizan controles inadecuados de reacción cruzada, cuando los antígenos son evaluados en áreas geográficas diferentes, cuando no son antígenos conservados en los genotipos de $T$. solium, cuando se evalúan en cerdos criados en confinamiento o criados al aire libre, cuando se presenta infección concomitante con gusanos adultos de T. solium, cuando los cisticercos tienen localizaciones diferentes (sistema nervioso central u otros tejidos) o cuando se usan para el diagnóstico de cisticercosis activa o calcificada, entre otros.

Finalmente, a la fecha, no se ha reportado un antígeno recombinante o sintético validado que pueda ser utilizado en una prueba sensible, específica y reproducible para el serodiagnósticos de la cisticercosis, pero, sin duda, el desarrollo y aplicación de pruebas de diagnóstico basadas en antígenos de "segunda generación", son una meta alcanzable, que contribuirá a:

1) mejorar la sensibilidad con que estos antígenos pueden ser reconocidos por los anticuerpos de pacientes con sospecha de cisticercosis, potenciando el paso de este criterio mayor a un criterio absoluto,

2) Ilenar los vacíos actuales en el conocimiento de la ecoepidemiología de esta parasitosis,

3) hacer un adecuado seguimiento de la respuesta inmune humoral en los proceso de vacunación,

4) mejorar el seguimiento al tratamiento farmacológico y

5) mejorar la supervisión de la infección humana durante y después de la intervención de una comunidad con los programas de educación y control.

\section{Lecturas recomendadas}

1. Centers for Disease Control and Prevention. Recommendation of the international task force for disease erradication. MMWR. 1993;42.

2. Deckers N, Dorny P. Immunodiagnosis of Taenia solium taeniosis/cysticercosis. Trends Parasitol. 2010;26:137-44.

3. Espíndola NM, Iha AH, Fernandes I, Takayanagui OM, Machado Ldos R, Livramento JÁ, et al. Cysticercosis immunodiagnosis using 18- and 14-kilodalton proteins from Taenia crassiceps cysticercus antigens obtained by immunoaffinity chromatography. J Clin Microbiol. 2005;43:3178-84.

4. Fleury A, Escobar A, Fragoso G, Sciutto E, Larralde C. Clinical heterogeneity of human neurocysticercosis results from complex interactions among parasite, 
host and environmental factors. Trans $\mathrm{R}$ Soc Trop Med Hyg. 2010;104:243-50

5. Flisser A, Gyorkos TW. Contribution of immunodiagnostic tests to epidemiological/ intervention studies of cysticercosis/taeniosis in México. Parasite Immunol. 2007;29:637-49.

6. Hernández M, Beltrán C, García E, Fragoso G, Gevorkian G, Fleury A, Parkhouse M, Harrison L, Sotelo J, Sciutto E. Cysticercosis: towards the design of a diagnostic kit based on synthetic peptides. Immunol Lett. 2000;71:13-7.

7. Ito A, Craig PS. Immunodiagnostic and molecular approaches for the detection of taeniid cestode infections. Trends Parasitol. 2003;19:377-81.

8. Lee YM, Handali S, Hancock K, Pattabhi S, Kovalenko VA, Levin A, et al. Serologic diagnosis of human Taenia solium cysticercosis by using recombinant and synthetic antigens in QuickELISATM. Am J Trop Med Hyg. 2011;84:587-93.
9. Organización Panamericana de la Salud. Epidemiología y control de la teniasis y cisticercosis en América Latina. Washington, D.C.: Organización Panamericana de la Salud: 1994.

10. Organización Panamericana de la Salud. Informe de la reunión de consulta de la OPS/OMS sobre el complejo teniasis/cisticercosis. Serie HCT/AIEPI, 5.E Washington, D.C.: Organización Panamericana de la Salud; 1997.

11. Praet N, Rodríguez-Hidalgo R, Speybroeck N, Ahounou S, Benítez-Ortiz W, Berkvens D, et al. Infection with versus exposure to Taenia solium: what do serological test results tell us? Am J Trop Med Hyg. 2010;83:413-5.

12. Tsang VC, Brand JA, Boyer AE. An enzyme-linked immunoelectrotransfer blot assay and glycoprotein antigens for diagnosing human cysticercosis (Taenia solium). J Infect Dis. 1989;159:50-9.

\title{
Evidencia de transmisión activa de cisticercosis por Taenia solium en comunidades rurales de Venezuela
}

\author{
María Milagros Cortez ${ }^{1}$, Cruz M. Aguilar ², Glenda Rojas ${ }^{1,3}$, Gonzalo Boggio4, María Lares \\ Elizabeth Ferrer ${ }^{1,5}$, Michael Parkhouse ${ }^{6}$ \\ 1 Instituto de Investigaciones Biomédicas, Facultad de Ciencias de la Salud, Sede Aragua, \\ Universidad de Carabobo \\ 2 Centro de Investigaciones en Enfermedades Tropicales, Facultad de Ciencias de la Salud, San \\ Carlos, Universidad de Carabobo \\ ${ }^{3}$ Departamento Clínico-Integral, Escuela de Bioanálisis, Facultad de Ciencias de la Salud, Sede \\ Aragua, Universidad de Carabobo. \\ ${ }^{4}$ Facultad de Ciencias Veterinarias, Universidad Central de Venezuela. \\ 5 Departamento de Parasitología, Escuela de Medicina, Facultad de Ciencias de la Salud, Sede \\ Aragua, Universidad de Carabobo. \\ ${ }^{6}$ Instituto Gulbenkian de Ciencia, Oeiras, Portugal
}

\section{Introducción}

El binomio teniasis/cisticercosis se refiere a las infecciones ocasionadas por Taenia solium, en sus estadios adulto (teniasis) y larvaria (cisticercosis). La cisticercosis ocurre en forma natural en porcinos y accidentalmente en humanos, por ingestión de los huevos infectantes expulsados con las heces de humanos portadores del gusano adulto.

Desde el punto de vista clínico, la manifestación más grave en el hombre es la neurocisticercosis que ocurre cuando la larva o metacestodo se localiza en el sistema nervioso central.

La cisticercosis es reconocida como un problema de salud pública en Latinoamérica $(1,2)$. Esta enfermedad es una evidencia inequívoca de las deficiencias socioeconómicas en una comunidad, pues prevalece en áreas donde coexisten los factores de riesgo asociados a la infección (escaso saneamiento ambiental, crianza empírica de cerdos, presencia de portadores del gusano adulto $T$. solium). Así que al resolver los problemas básicos de saneamiento, no sólo se controlaría la teniasis/cisticercosis, sino que habría beneficios colaterales por disminución de otras infecciones ligadas a la contaminación fecal $(3,4)$.

Aunque la teniasis/cisticercosis se ha demostrado en países vecinos, en Venezuela, sin embargo, existe poca información oficial sobre la prevalencia o sobre el impacto de la cisticercosis en humanos o porcinos, posiblemente, por subregistro dadas las limitaciones en su diagnóstico $(5,6)$. El presente trabajo aborda el estudio seroepidemiológico del binomio teniasis/cisticercosis en tres comunidades rurales de Venezuela.

\section{Materiales y métodos}

El estudio se enfocó, en primer lugar, en la evaluación serológica de cisticercosis en humanos y porcinos por detección de anticuerpos anti- 
metacestodos de Taenia sp. por ensayos de ELISA (7), utilizando fluido vesicular de metacestodos obtenidos de cerdo naturalmente infectado, $y$, en segundo lugar, antígenos circulantes de metacestodos por prueba de ELISA de captura basada en el anticuerpo monoclonal HP-10 (8).

El segundo aspecto considerado fue la detección de portadores del gusano adulto de $T$. solium en muestras de heces, por coprología.

Finalmente, la presencia de factores de riesgo para la teniasis/cisticercosis se evaluó por observación directa (crianza de cerdos, acceso a agua potable, presencia de letrinas) y por encuestas individuales (hábitos de defecación, ingestión de cerdo infectado, expulsión de proglótidos, entre otros). Los pacientes portadores del gusano adulto fueron tratados para la eliminación del parásito.

\section{Resultados}

Los resultados expresados en la tabla 1 indican porcentajes de seropositivos para antígenos y anticuerpos anti-metacestodos de Taenia sp., tanto en porcinos como en humanos, lo que sugiere: a) presencia de individuos con infección activa de cisticercosis (positivos a antígenos de metacestodos de Taenia sp.) y b) exposición al parásito (positivos a anticuerpos). Además, en las comunidades Potrero Largo y Valle del Río se logró identificar portadores de $T$. solium, y en esta última existe un paciente con diagnóstico clínico y radiológico de neurocisticercosis.

La tabla 2 relaciona los factores de riesgo asociados a la infección por teniasis/cisticercosis encontrados en las comunidades estudiadas resaltando la crianza de cerdos que deambulan en búsqueda de alimento, y el libre acceso a las heces humanas, por el hábito humano de defecar en el medio ambiente, dos condiciones básicas para el mantenimiento del ciclo de vida de T. solium (4). Si se suma la presencia demostrada de portadores de $T$. solium en estas comunidades, se tienen las condiciones suficientes para el establecimiento y el mantenimiento del binomio teniasis/cisticercosis como endemias en estas comunidades $(3,9)$.

En conclusión, las evidencias serológicas y epidemiológicas presentadas aquí ponen de manifiesto focos de transmisión de teniasis/ cisticercosis en zonas rurales de Venezuela.

Tabla 1. Transmisión de teniasis/cisticercosis en comunidades rurales venezolanas.

\begin{tabular}{|c|c|c|c|c|c|c|c|}
\hline \multirow[b]{2}{*}{ Comunidades } & \multicolumn{3}{|c|}{ Porcinos } & \multicolumn{3}{|c|}{ Humanos } & \multirow[b]{2}{*}{ NCC } \\
\hline & AG & AC & Metacestodos & AG & $A C$ & $\begin{array}{l}\text { Taenia sp. } \\
\text { ad. }\end{array}$ & \\
\hline La Yuca, Estado Yaracuy & 42,3 & 65,4 & + & ND & 34,7 & 1,06 & ND \\
\hline Potrero Largo, Estado Cojedes & 13,0 & 60,4 & ND & 8,7 & 13,7 & $0,7^{*}$ & ND \\
\hline Valle del Río, Estado Cojedes & 26,0 & 32,0 & ND & 47,0 & 37,5 & $1,3^{*}$ & 0,74 \\
\hline
\end{tabular}

AG: porcentaje de individuos positivos a antígenos circulantes de metacestodos de Taenia sp. detectados por HP-10 ELISA

AC: porcentaje de individuos positivos a anticuerpos anti-metacestodos de Taenia solium, detectados por ELISA, fluido vesicular

Metacestodos: detección por biopsia de metacestodos de Taenia solium en porcinos

Taenia sp. ad.: porcentaje de individuos con expulsión de Taenia sp. adulto en la comunidad

* En estos individuos se logró la identificación de Taenia solium.

NCC: porcentaje de individuos con neurocisticercosis clínica y radiológicamente demostrada.

ND: sin determinar

Tabla 2. Presencia de factores de riesgo para el binomio teniasis/cisticercosis en comunidades rurales. Venezuela.

\begin{tabular}{|c|c|c|c|c|c|c|}
\hline & Analfabetismo & $\begin{array}{c}\text { Defecación en } \\
\text { el medio } \\
\text { ambiente }\end{array}$ & $\begin{array}{l}\text { Individuos } \\
\text { parasitados* }\end{array}$ & $\begin{array}{l}\text { Crianza de } \\
\text { cerdos libres }\end{array}$ & $\begin{array}{c}\text { Acceso agua } \\
\text { potable }\end{array}$ & $\begin{array}{c}\text { Recolección } \\
\text { de desechos } \\
\text { sólidos }\end{array}$ \\
\hline La Yuca, Estado Yaracuy & ND & 93,4 & 77,1 & 100 & Parcial & NO \\
\hline $\begin{array}{l}\text { Valle del Río, } \\
\text { Estado Cojedes }\end{array}$ & 14,9 & 63,7 & 84,2 & 77,4 & Parcial & NO \\
\hline $\begin{array}{l}\text { Potrero Largo, } \\
\text { Estado Cojedes }\end{array}$ & 11,8 & 60,9 & 83,5 & 14,8 & Parcial & NO \\
\hline
\end{tabular}

*Parasitosis intestinales: ascaridiasis, anquilostomiasis, tricuriasis, amibiasis, giardiasis 
Finalmente, consideramos al conjunto de medidas involucradas en este estudio (diagnóstico, tratamiento, educación) de teniasis/cisticercosis como plan piloto aplicables hacia el conocimiento y control de estas parasitosis en otras comunidades de nuestro país.

\section{Referencias}

1. Engels D, Urbani C, Belotto A, Meslin F. Savioli L. The control of human (neuro)cysticercosis: which way forward?. Acta Trop. 2003;87:177-82.

2. García-Noval J, Allan J, Fletes C, Moreno E, De Mata F, Torres-Álvarez R, et al. Epidemiology of Taenia solium taeniasis and cysticercosis in two rural Guatemalan communities. Am J Trop Med Hyg. 1996;55:282-9.

3. Schantz PM, Cruz M, Sarti E, Pawlowski Z. Potential eradicability of taeniasis and cysticercosis. Bull PAHO. 1993;27:397-403.

4. Sciutto E, Fragoso G, Fleury A, Laclette JP, Sotelo J Aluja A, et al. Taenia solium disease in humans and pigs: an ancient parasitosis disease rooted in developing countries and emerging as a major health problem of global dimensions. Microb Infect. 2000;2:1875-90.
5. Cortez MM, Boggio G, Guerra ML, Rodríguez M, Rojas $\mathrm{G}$, Ferrer $\mathrm{E}$, et al. Evidence that active transmission of porcine cysticercosis occurs in Venezuela. Trop Anim Health Prod. 2010;42:531-7.

6. Alarcón B, Colmenares C. Las limitaciones del diagnóstico de la cisticercosis humana en Venezuela. Fecha de consulta: 2 de junio de 2011.Disponible en: http://caibco.ucv.ve/caibco/vitae/VitaeOnce/Articulos/ MedicinaTropical/ArchivosHTML/MediciTropi.pdf 2002.

7. Larralde C, Laclette JP, Owen CS, Madrazo I, Sandoval M, Bojalil R, et al., Reliable serology of Taenia solium cysticercosis with antigens from cyst vesicular fluid: ELISA and hemaglutination test. Am J Trop Med Hyg. 1989;35:965-73.

8. Harrison L, Joshua G, Wright S, Parkhouse RME. Specific detection of circulating surface-secreted glycoproteins of viable cysticerci in $T$. saginata cysticercosis. Parasit Immunol.1989;11:351-70.

9. Sarti E, Schantz PM, Plancarte A, Wilson M, Gutierrez IO, López AS, et al. Prevalence and risk factors for Taenia solium taeniasis an cysticercosis in humans and pigs in a village in Morelos, Mexico. Am J Trop Med Hyg. 1992;46:677-85.

\title{
Cisticercosis, un obstáculo para el desarrollo: la situación en Brasil
}

\author{
Marcello O. Sato \\ Biotério e Laboratório de Experimentação Animal, Escola de Medicina, \\ Universidade Federal do Tocantins, Palmas, Brasil
}

La cisticercosis es una enfermedad zoonótica causada por la presencia de metacestodos de Taenia saginata y Taenia solium en los tejidos de sus huéspedes intermediarios (ganado bovino y porcino) y, ocasionalmente, en el hombre. La infección se produce por el consumo de alimentos contaminados con huevos de Taenia sp.; después de ser ingeridos, en el cuerpo se desarrollan como cisticercos.

El complejo teniasis-cisticercosis es un problema importante de salud pública; el hombre actúa como huésped intermediario accidental que conduce al desarrollo de los cisticercos en sus tejidos, especialmente en el sistema nervioso central. La neurocisticercosis es una enfermedad que con mayor frecuencia afecta el sistema nervioso central (Albuquerque y Gallardo, 1995). Los principales agravantes de esta enfermedad son las malas condiciones de salud y los problemas económicos y sociales, junto con la cultura local que contribuyen a la propagación de la misma (Takayanagui y Leite, 2001).

Un factor que aumenta el número de casos de cisticercosis humana es la matanza clandestina, que ha elevado el porcentaje, según las autoridades federales. El número de consumidores de carne clandestina o no inspeccionada, es bastante alto debido a la disminución de costos en comparación con la carne inspeccionada o legalizada. A menudo, este hábito se da por la propia cultura local, pueden contribuir a la alta incidencia de la enfermedad, ya que la carne es el vehículo de la misma contaminación (Fundação Nacional da Saúde, 1996).

En Brasil, la neurocisticercosis se encuentra con frecuencia en los estados de São Paulo, Minas Gerais, Paraná y Goiás (Takayanagui y Leite, 2001). Sin embargo, el porcentage de enfermos con neurocisticercosis no se conoce debido a la falta de registro de la enfermedad en la mayoría de los municipios.

El estado de Paraná es uno de los principales focos de la neurocisticercosis en Brasil. Según el Ministerio de Salud de Paraná, se registraron 4.040 casos de neurocisticercosis humana, entre los años 1980 y 2000, las personas entre 30 y 49 años tuvo un índice mayor de esta enfermedad, con 419 (39,3\%) casos. A continuación se indica el número de casos de neurocisticercosis que 
se presentaron entre los años 1993 y 2000 en el estado de Paraná.

La región de Ribeirão Preto, en 1983, fue considerada una zona endémica de cisticercosis, lo que representa el $7,5 \%$ de las hospitalizaciones en el Servicio de Neurología del Hospital de las Clínicas de la Universidade de São Paulo (Takayanagui, et al., 1996). Como resultado de esto se creó un programa para controlar la enfermedad, pasando ahora a ser registrado obligatoriamente.

Según Clemente (1990), el estado de Rio de Janeiro también presentó casos entre los años 1981 y 1989, siendo más de 100 el número de nuevos casos de neurocisticercosis; estos casos se asociaron con las malas condiciones sanitarias y nivel socioeconómico de la región. En 1996, Takayanagui, et al., encontraron que la enfermedad había empeorado en la región, pues $21 \%$ de los casos denunciados tenía la forma activa, es decir, con cisticercos en el parénquima cerebral. En 2005, Mendes, et al., analizaron tomografías computarizadas de 36.379 individuos del estado de Rio de Janeiro, donde se diagnosticaron 72 casos de la parasitosis, la mayoría de ellos en mujeres $(62,5 \%)$, y $93,1 \%$ de los casos eran de fase crónica. Esta enfermedad sigue siendo común en las áreas metropolitanas, con tasas más altas de casos cada año.

Con los estudios realizados por varios autores, Cruz, et al. (1995) describió la presencia de cisticercosis de los datos obtenidos en Brasil entre los años 1916 y 1991, y se puede hacer un análisis de la evolución del número mínimo de casos en las principales áreas. Aunque sean utilizados diferentes métodos de diagnóstico, se puede observar que la enfermedad siempre se mantiene. Sato, et al. (2006) confirmaron la presencia y la transmisión activa del complejo teniasis-cisticercosis en el centro-norte de Brasil en muestras de quistes obtenidos de cerdos, y reveló la presencia del genotipo afroamericano de $T$. solium en las personas y en pruebas de ELISA con antígenos nativos y recombinantes se reveló la presencia de la cisticercosis humana.

El Serviço de Inspeção Federal (SIF) es el órgano de supervisión federal brasilero responsable de la supervisión de los productos de origen animal, también es el órgano oficial de información sobre las enfermedades de importancia en salud pública. Sin embargo, por el hecho de no trabajar de forma adecuada sus datos, acaban perdiéndose, cuando muy bien podrían ser utilizados como una ayuda en los programas de prevención y control de las zoonosis más frecuente en el país.

En un estudio realizado en el estado de Rio de Janeiro, de 494.620 animales sacrificados de 38 municipios, 9.656 (1,95\%) tenían cisticercos.

Se adelantó un estudio sobre la presencia de cisticercos en vivo $(0,8-1 \%)$ y calcificados $(99,0 \%)$ en el período comprendido entre 1997 y 2003. La ciudad de Duas Barras presentó la mayor tasa de parasitismo en los animales, con una media total de 118 animales sacrificados por año y un promedio de $4,29 \%$ con cisticercosis (Pereira, 2006). Estos datos podrán ser utilizados como una ayuda en la cartografía de la enfermedad, contribuyendo a la labor epidemiológica.

Los valores de prevalencia de esta enfermedad en Brasil son difíciles de obtener, ya que los estudios se realizan en diferentes regiones y con metodologías diversas, al no existir un protocolo y, por lo tanto, no se pueden comparar los datos entre las regiones. Sin embargo, los estudios clínicos y de autopsia, junto con los informes de casos, la epidemiología de las evaluaciones y los datos de los mataderos bajo control del Servicio Federal de Inspección, permite una noción de las regiones más afectadas y el grado de endemia de una población dada, los datos esencial para quienes planean un programa de control y erradicación. Por otra parte, la presentación obligatoria de informes de diagnóstico precoz podría ayudar a alertar la vigilancia epidemiológica en las zonas endémicas.

\section{Referencias}

1. Albuquerque ES, Galhardo I. Neurocisticercose no Estado do Rio Grande do Norte - relato de oito casos. Arq Neuropsiquiatr. 1995;53:464-70.

2. Clemente $H$, WemeckA. Neurocisticercose: incidência no Estado do Rio de Janeiro. Arq Neuropsiquiatr. 1990;48:207-9.

3. Costa-Cruz JM, Rocha A, Silva AM, et al. Ocorrência de cisticercose em necropsias realizadas em Uberlância, Minas Gerais, Brasil. Arq Neuropsiquiatr. 1995;53:227-32.

4. Fundação Nacional da Saúde. Projeto para o controle do complexo teníase/cisticercose no Brasil. Brasília: FUNASA; 1996.

5. Pereira MAVC, Schwanz VS, Barbosa CG. Prevalência da cisticercose em carcaças de bovinos abatidos em matadouros-frigoríficos do estado do Rio de Janeiro, submetidos ao controle do Serviço de Inspeção Federal (SIF-RJ), no período de 1997 a 2003. Arq Inst Biol. (São Paulo) 2006;73:83-7.

6. Sato MO, Cavalcante TV, Sako Y, Nakao M, Yamasaki $\mathrm{H}$, Yatsuda AP, et al. Evidence and potential for transmission of human and swine Taenia solium cysticercosis in the Piracuruca region, Piauí, Brazil. Am J Trop Med Hyg. 2006;75:933-5. 
7. Spina-França A, Livramento JA, Machado LR. Cysticercosis of the central nervous system and cerebrospinal fluid immunodiagnosis of 1573 patients in 63 years (1929-1992). Arq Neuropsiquiatr. 1993;51:16-20.

8. Takayanagui OM, Jardim E. Aspectos clínicos da neurocisticercose: análise de 500 casos. Arq
Neuropsiquiatr. 1983;41:50-63.

9. Takayanagui OM, Leite JP. Neurocisticercose. Rev Soc Bras Med Trop. 2001;34:283-90.

10. Ungar ML, Germano PML. Prevalência da cisticercose bovina no Estado de São Paulo (Brasil). Rev Saúde Públ.1992;26:167-72.

\title{
Epidemiología de la infección por Taenia solium en México
}

\author{
A. Fleury ${ }^{1,2}$, E. Sciutto ${ }^{1}$, A. de Aluja ${ }^{3}$, N. Villalobos ${ }^{3}$, J. Morales ${ }^{1}$, C. Larralde ${ }^{1}$, J. J. Martínez ${ }^{3}$ \\ ${ }^{1}$ Instituto de Investigaciones Biomédicas, Universidad Nacional Autónoma de México, México, D.F., \\ México \\ 2 Instituto Nacional de Neurología y Neurocirugía, SSA, México, D.F., México \\ Facultad de Medicina Veterinaria y Zootecnia, Universidad Nacional Autónoma de México, México, \\ D.F., México
}

Las características de las enfermedades causadas por Taenia solium dificultan la obtención de datos fehacientes sobre su epidemiología. Tanto la teniasis (infección intestinal por el parásito adulto) como la cisticercosis (infección por el parásito larvario, generalmente en el sistema nervioso central) son frecuentemente asintomáticas, lo que resulta en un subregistro de los casos. Además, el acceso limitado de la población a los estudios neurorradiológicos [tomografías computadorizadas (TC) y resonancia magnética (RM)], pruebas hasta la fecha más certeras para el diagnóstico de la neurocisticercosis, dificulta aún más establecer estadísticas confiables.

La herramienta diagnóstica más utilizada para la cisticercosis porcina, el examen de la lengua, requiere personal bien entrenado, es traumática para el cerdo y su sensibilidad y especificidad no son óptimas. Pocos estudios han establecido el diagnóstico de cisticercosis porcina con base en la necropsia del animal que es el método definitivo para este propósito.

Debido a estas razones, los datos actualmente disponibles sólo son una estimación del estado de la teniasis-cisticercosis en México y, probablemente, subvaloran el impacto de esta parasitosis.

Cabe recordar que en México una norma oficial emitida en 1994 y modificada en 2004, insta a todo el personal de salud a reportar los casos de teniasis y cisticercosis (1). Estos datos muestran una disminución muy pronunciada de los casos de ambas infecciones (2). Infortunadamente, la confiabilidad de esta información dista de lo esperado, como se reporta a continuación.

\section{Teniasis}

Se han realizado diferentes estudios, principalmente en comunidades rurales 0 en diferentes poblaciones específicas (expendedores de alimentos, soldados, mercados) (3-6). Los distintos estudios concuerdan con una prevalencia medida por coproantígenos de alrededor del $1 \%$. Lamentablemente, pocos estudios han confirmado este resultado mediante el tratamiento del paciente y la recuperación del parásito adulto. En los estudios en los que se ha realizado este procedimiento, en pocos casos se recupera el parásito. Esta situación puede resultar tanto de la baja especificidad del ensayo de coproantígeno como de la vulnerabilidad del gusano adulto a las modificaciones ambientales del intestino que provoca su espontánea expulsión.

\section{Cisticercosis porcina}

Se estima que la población de cerdos en México es de más de 14'000.000 individuos, de los cuales, entre 30 y $40 \%$ son criados en condiciones no tecnificadas (7). Es una práctica frecuente en las comunidades rurales que los cerdos deambulen en las calles en búsqueda de alimento, en particular, la materia fecal humana. El consumo de estos animales se realiza, generalmente, durante fiestas comunitarias y sin inspección sanitaria. Los estudios recientes realizados en cerdos de traspatio de comunidades rurales del centro del país muestran la persistencia de la cisticercosis porcina. Utilizando el método de diagnóstico del examen de lengua, se han reportado prevalencias hasta de $33 \%$ (8). Un estudio hecho en 33 comunidades del estado de Morelos entre 2002 y 2003, reportó en 15 de ellas prevalencias de cisticercosis porcina superiores a $15 \%(1.747$ animales examinados) (9). Los estudios basados en la inspección de carne de un muestreo de los cerdos sacrificados en 2006 encontró una prevalencia de 19,6\% (40/204) (10). 


\section{Neurocisticercosis humana}

En dos estudios epidemiológicos realizados en 2003 y 2006 en comunidades rurales del centro de México $(11,12)$ utilizando TC $(n=859)$ en una muestra representativa en edad y sexo de la población, se detectaron entre el 9 y el $10 \%$ de lesiones compatibles con neurocisticercosis. La mayoría eran calcificaciones únicas, aunque se encontraron calcificaciones múltiples y algunos con formas vesiculares. La mayor parte de los casos detectados fueron asintomáticos.

A nivel hospitalario, recientemente, se evaluó la variación del número de casos atendidos en el Instituto Nacional de Neurología y Neurocirugía entre 1994 y 2009 (13). Esta institución, localizada en la capital del país, recibe pacientes adultos de todo el país que no tienen seguro social. Dos parámetros fueron estudiados: el número total de pacientes atendidos (consultas y hospitalización) en 1994 y 2004 y la evolución del número de pacientes hospitalizados anualmente con este diagnóstico entre 1995 y 2009. No se encontraron diferencias significativas en la proporción de pacientes atendidos con este diagnóstico entre $1994(100 / 4098,2,4 \%)$ y 2004 (120/4706, $2,5 \%)$, $p=0,74$. La tendencia anual de hospitalización en el Servicio de Neurología fue estable entre 1995 y $2009(r=0,12, p=0,67)$ mientras que se observó una disminución significativa en el servicio de neurocirugía $(r=-0.79, P=0.001)$. Esta disminución está probablemente relacionada con el diagnóstico más eficiente de los pacientes así como con el tratamiento más temprano.

\section{Conclusiones}

Apesar del optimismo de las estadísticas oficiales, los datos reportados muestran una persistencia en México de los casos de infecciones por el parásito $T$. solium tanto en el humano como en el cerdo. Los datos reportados en este resumen nos permiten sostener que la neurocisticercosis sigue siendo una enfermedad prevalente en México, causa de morbilidad en individuos jóvenes a los que afecta gravemente en su calidad de vida (14).

Es por estas razones que deben continuarse los esfuerzos para su control y eventual erradicación. Contamos para este propósito herramientas que se han desarrollado para su diagnóstico y prevención y que son el resultado de años de investigación de múltiples grupos de estudio en cisticercosis en México $(10,15)$.

\section{Referencias}

1. Secretaría de Salud. Modificación a la Norma Oficial Mexicana NOM-021-SSA2-1994, para la prevención y control del complejo teniosis/cisticercosis en el primer nivel de atención médica. Diario Oficial de la Federación, 21 mayo de 2004. Disponible en: http:// www.salud.gob.mx/unidades/cdi/nom/m021ssa294. html.

2. Flisser A, Correa D. Neurocysticercosis may no longer be a public health problem in Mexico. PLoS Negl Trop Dis. 2010;4:e831.

3. Barton Behravesh C, Mayberry LF, Bristol JR, Cardenas VM, Mena KD, Martínez-Ocaña J, Flisser A, Snowden KF. Population-based survey of taeniasis along the United States-Mexico border. Ann Trop Med Parasitol. 2008;102:325-33.

4. Sarti E, Schantz PM, Avila G, Ambrosio J, MedinaSantillán R, Flisser A. Mass treatment against human taeniasis for the control of cysticercosis: a populationbased intervention study. Trans R Soc Trop Med Hyg. 2000;94:85-9.

5. Martínez-Maya JJ, de Aluja AS, Ávila-Ramírez G, Aguilar-Vega L, Plancarte-Crespo A, JaramilloArango CJ. Taeniasis and detection of antibodies against cysticercus among inhabitants of a rural community in Guerrero State, México. Salud Pública Méx. 2003;45:84-9.

6. García-García ML, Torres M, Correa D, Flisser A, Sosa-Lechuga A, Velasco O, Meza-Lucas A, et al. Prevalence and risk of cysticercosis and taeniasis in an urban population of soldiers and their relatives. Am J Trop Med Hyg. 1999;61:386-9.

7. de Aluja A. La cisticercosis porcina en México. En: Cisticercosis: guía para profesionales de la salud. Biblioteca de la Salud. México: Fondo de Cultura Económico; 2006. p. 104-32.

8. Morales J, Velasco T, Tovar V, Fragoso G, Fleury A, Beltrán $\mathrm{C}$, et al.. Castration and pregnancy of rural pigs significantly increase the prevalence of naturally acquired Taenia solium cysticercosis. Vet Parasitol. 2002;108:41-8.

9. Morales J, Martínez,JJ, García-Castella J, Peña N, Maza V, de Aluja N, et al. Taenia solium: the complex interactions of biological, social, geographical and commercial factors, involved in the transmission dynamics of pig cysticercosis in highly endemic areas. Ann Trop Med Parasitol. 2006;100:123-35.

10. Morales J, de Aluja A, Martínez JJ, Hernández M, Rosas G, Villalobos N, et al. Recombinant S3Pvacphage anticysticercosis vaccine; Simultaneous protection against cysticercosis and hydatid disease in rural pigs. Vet Parasitol. 2011;176:53-8.

11. Fleury A, Gómez T, Álvarez I, Meza D, Huerta M, Chavarría $A$, et al. High prevalence of calcified silent neurocysticercosis in a rural village of México. Neuroepidemiology. 2003;22:139-45.

12. Fleury A, Morales J, Bobes RJ, Dumas M, Yánez $\mathrm{O}$, Piña J, et al. An epidemiological study of familial neurocysticercosis in an endemic Mexican community. Trans R Soc Trop Med Hyg. 2006;100:551-8. 
13. Fleury A, Moreno García J, Valdez Aguerrebere $P$, de Sayve Durán M, Becerril Rodríguez P, Larralde C, Sciutto E. Neurocysticercosis, a persisting health problem in Mexico. PLoS Negl Trop Dis. 2010;4:e805.

14. Bhattarai R, Budke CM, Carabin H, Proaño JV, FloresRivera J, Corona T, et al. Quality of life in patients with neurocysticercosis in Mexico. Am J Trop Med Hyg. 2011;84:782-6.

15. Hernández M, Cervantes J, Cabrera-Ponce JL, Herrera-Estrella L, Rosas Salgado G, Villarreal ML, et al. Development of an oral anti-cysticercosis vaccine delivered in genetically modified embryogenic callus of papaya. Transgenic Plant J. 2011; en prensa.

\title{
Avances en la optimización de la vacuna S3Pvac contra cisticercosis porcina y experiencias en su aplicación en un programa regional de control de teniasis-cisticercosis
}

\author{
E. Sciutto', G. Fragoso', J. Morales², N. Villalobos², M. Hernández', J. Cervantes ${ }^{1}$, A. Blancas ${ }^{1}$, I. \\ Flores $^{4}$, G. Rosas 3 , M. Betancourt ${ }^{4}$, S. Rosales ${ }^{5}$, A. Fleury ${ }^{1}$, J. J. Martínez ${ }^{2}$, C. Larralde ${ }^{1}$, A. Aluja ${ }^{2}$ \\ 1 Instituto de Investigaciones Biológicas, México, D.F., México \\ 2 Facultad de Medicina Veterinaria y Zootecnia, Universidad Nacional Autónoma de México, México, \\ D.F., México \\ ${ }^{3}$ Facultad de Medicina, Universidad Nacional Autónoma de México, México, D.F., México \\ 4 Facultad de Ciencias Agropecuarias, Universidad Autónoma de Morelos, Morelos, México \\ ${ }^{5}$ Facultad de Ciencias Químicas, Universidad Autónoma de San Luis de Potosí, San Luis de Potosí, \\ México.
}

En México, la cisticercosis continúa afectando la salud humana (1) y prevalecen las condiciones que propician su transmisión: $56 \%$ de los cerdos se producen en condiciones semitecnificadas o de traspatio (aproximadamente, 7'000.000 de cerdos que en su mayoría se renuevan anualmente (2); ha disminuido el ingreso per cápita de la población de México de bajos ingresos y de la población rural que representa el $60 \%$ y el $24 \%$ del total de la población, respectivamente $(3,4)$; continúa el consumo de carne sin inspección sanitaria y la falta de servicios sanitarios adecuados en el medio rural.

Considerando esta realidad, en conjunto con la Secretaría de Agricultura, Ganadería, Desarrollo Rural, Pesca y Alimentación (SAGARPA) iniciamos en septiembre de 2009 un programa de control regional contra la teniasis-cisticercosis que incorpora la vacunación de cerdos de traspatio y promover el conocimiento de la enfermedad y los factores que propician su transmisión, que se planea extender nacionalmente con el propósito de prevenir $y$, eventualmente, erradicar esta parasitosis en México (5).

La cisticercosis porcina, fase indispensable para la transmisión de la cisticercosis, es prevenible por vacunación y ofrece así un blanco idóneo en el cual incidir para interrumpir el ciclo de transmisión de la teniasis-cisticercosis por Taenia solium.

Las vacunas contra cisticercosis porcina, constituidas por antígenos nativos, sintéticos o recombinantes han demostrado eficiencia de protección en condiciones naturales de transmisión (6-9). En nuestro grupo de investigación desarrollamos una vacuna sintética, multivalente y multiestadio contra cisticercosis porcina, denominada S3Pvac, constituida por tres péptidos de 18, 12 y 8 aminoácidos denominados GK1, KETc1 y KETc12, respectivamente (7). GK1 fue deducido a partir del antígeno protector denominado KETc7 de 100 aminoácidos (10). Los componentes de S3Pvac fueron seleccionados por su capacidad protectora en contra de la cisticercosis en ratón por Taenia crassiceps (10). Los tres péptidos, originalmente identificados en una biblioteca de cDNA de $T$. crassiceps, resultaron ser compartidos por T. solium, Taenia saginata, Echinococcus granulosus y Echinococcus multilocularis y se expresan en las tres fases del parásito (tenia, oncosfera, cisticerco) (11).

S3Pvac demostró proteger totalmente al $50 \%$ de los cerdos vacunados y reducir en $98 \%$ la cantidad de cisticercos en un ensayo de campo (2) realizado con 240 cerdos (120 vacunados/120 controles) (7). Con el propósito de optimizar su producción masiva a bajos costos, S3Pvac fue expresada fusionando las secuencias que codifican para los péptidos de S3Pvac y el antígeno KETc7 a los genes que codifican las proteínas repetidas de la superficie del fago filamentoso M13 (12). Este sistema, además de expresar repetidamente los péptidos de la vacuna en cada partícula de fago, no requiere el uso de adyuvante para generar inmunidad y puede ser producido masivamente en biorreactores. 
La nueva versión de la vacuna, denominada S3Pvac-fago, demostró proteger totalmente del 54 al $62 \%$ los cerdos vacunados y en $87 \%$ la cantidad de cisticercos establecidos $(8,13)$ en un ensayo de campo que incluyó la revisión por necropsias de 391 cerdos (187 vacunados/204 controles). En este mismo estudio, S3Pvac-fago resultó reducir en $56 \%$ la prevalencia de hidatidosis (13). S3Pvac-fago se está aplicando en miles de dosis en el marco programa de control en desarrollo del Estado de Guerrero desde septiembre de 2009 con resultados que indican la efectividad de esta intervención (tabla 1).

Con el propósito de desarrollar una versión oral, se ha producido una tercera generación de S3Pvac expresada en el genoma de callos de papaya (14). S3Pvac-papaya ha demostrado alta eficiencia administrada oralmente en contra de la cisticercosis de ratón por $T$. crassiceps y de la cisticercosis en conejos por Taenia pisiformis (14, 15) sin el uso de adyuvantes. Esta vacuna se está evaluando actualmente en cerdos para recomendar su aplicación masiva.

Con el fin de aumentar la eficiencia de S3Pvacfago agregamos el antígeno protector HP6/ TSOL18 expresado en M13, cuya eficiencia ha sido recientemente demostrada en un ensayo de protección realizado en campo (9). La vacuna S3Pvac-fago más HP6/TSOL18-M13, constituye la nueva generación de vacuna inyectable denominada S4Pvac-fago. Esta vacuna se encuentra actualmente en evaluación.

Simultáneamente, se está optimizando la vacuna S3Pvac-papaya debido a que, a pesar de poseer una alta capacidad protectora, el bajo nivel de expresión de los péptidos de la vacuna limita su producción masiva. Para la optimización de la vacuna oral se están expresando los péptidos que constituyen la vacuna S3Pvac-papaya y el antígeno HP6/TSOL18 en plantas "transplastómicas" de papaya y lechuga, para generar la vacuna S4Pvacpapaya/lechuga.

Estos sistemas "transplastómicos" se han empleado extensa y eficientemente para la síntesis de biofarmacéuticos funcionales con altos rendimientos y permitirá la producción masiva de la vacuna S4Pvac con alta eficiencia y a bajos costos en un tipo de biomasa idóneo para su aplicación oral.

Cabe mencionar que el disponer de una vacuna oral nos permitiría entregarla a los propietarios de los cerdos para su administración cuando los alimentan lo que reduciría críticamente las dificultades logísticas y los costos de aplicación que conlleva el uso de la vacuna parenteral en cerdos de traspatio y permitiría extender el programa de control a nivel nacional.

\section{Referencias}

1. Fleury A, Moreno García J, Valdez Aguerrebere P, de Sayve Durán M, Becerril Rodríguez P, Larralde C, et al. Neurocysticercosis, a persisting health in Mexico. PLoS Negl Trop Dis. 2010;4:e805.

2. SIAP-SAGARPA. Estadísticas sobre la producción porcina para los años 2008 y 2009. Fecha de consulta: abril de 2010. Disponible en: http://siap.gob.mx.

3. INEGI. Encuesta Nacional de Ingresos y Gastos de los Hogares (ENIGH), 2008. Disponible en: http:// www.inegi.org.mx.

4. INEGI. II Conteo de Población y Vivienda 2005. Disponible en: http://www.inegi.org.mx.

5. Olguín S. Programa de control de la teniasiscisticercosis. Gaceta Biomédica. 2010;15:8-9. Disponible en: http://www.biomedicas.unam.mx/ buscar_noticias/gaceta_biomedicas.html.

6. Molinari JL, Rodríguez D, Tato P, Soto R, Arechavaleta F, Solano S. Field trial for reducing porcine Taenia solium cysticercosis in Mexico by systematic vaccination of pigs. Vet Parasitol. 1997;69:55-63.

Table 1. Summary of our experiences published in vaccination against porcine cysticercosis.

\begin{tabular}{|c|c|c|c|c|c|c|}
\hline \multirow[t]{2}{*}{ Vaccine employed } & \multicolumn{3}{|c|}{ Experimental } & \multicolumn{3}{|c|}{ Field } \\
\hline & $\% E f 1$ & $\% E f 2$ & \# of pigs & $\% E f 1$ & $\% E f 2$ & \# of pigs \\
\hline T. crassiceps $\left(T_{a q}\right)$ & $0-58$ & $0-28$ & 89 & & ND & \\
\hline Native purified antigens & 98 & $83 \%$ & 12 & & ND & \\
\hline ‡S3Pvac synthetic & & ND & & 99 & 50 & 240 \\
\hline S3Pvac- phage & $49-83$ & $0-33$ & 11 & 87 & $54-62$ & 391 \\
\hline Total & & & 112 & & & 631 \\
\hline
\end{tabular}

† Number of animals in each row including vaccinated and the controls pigs

$\%$ Ef1: percent of the reduction in the parasite load

$\%$ Ef2: percent of pigs totally protected

‡ Includes experiments performed using each of the S3Pvac versions

$\mathrm{T}_{\mathrm{ag}}$ : total antigens; ND: not determined 
7. Huerta M, de Aluja AS, Fragoso G, Toledo A, Villalobos $N$, Hernandez $M$, et al. Synthetic peptide vaccine against Taenia solium pig cysticercosis: successful vaccination in a controlled field trial in rural Mexico. Vaccine. 2001;20:262-6.

8. Morales J, Martínez JJ, Manoutcharian K, Hernández M, Fleury A, Gevorkian G, et al. Inexpensive anticysticercosis vaccine: S3Pvac expressed in heat inactivated M13 filamentous phage proves effective against naturally acquired Taenia solium porcine cysticercosis. Vaccine. 2008;26:2899-905.

9. Assana E, Kyngdon CT, Gauci CG, Geerts S, Dorny $\mathrm{P}$, De Deken R, et al. Elimination of Taenia solium transmission to pigs in a field trial of the TSOL18 vaccine in Cameroon. Int J Parasitol. 2010;40:515-9.

10. Manoutcharian K, Rosas G, Hernández M, Fragoso $\mathrm{G}$, Aluja A, Villalobos $\mathrm{N}$, et al. Cysticercosis: identification and cloning of protective recombinant antigens. J Parasitol. 1996;82:250-4.

11. Rassy D, Bobes RJ, Rosas G, Anaya VH, Brehm K, Hernández B, et al. Characterization S3Pvac anticysticercosis vaccine components: implications for the development of an anti- cestodiasis vaccine. PLoS On. 2010;5:e11287.

12. Manoutcharian K, Díaz-Orea A, Gevorkian G, Fragoso G, Acero G, Gonzalez E, et al. Recombinant bacteriophage-based multiepitope vaccine against Taenia solium pig cysticercosis. Vet Immunol Immunopathol. 2004;99:11-24.

13. Morales J, de Aluja AS, Martínez JJ, Hernández M, Rosas G, Villalobos N, et al. Recombinant S3Pvacphage anticysticercosis vaccine: Simultaneous protection against cysticercosis and hydatid disease in rural pigs. Vet Parasitol. 2011;176:53-8.

14. Hernández M, Cabrera-Ponce JL, Fragoso G, LópezCasillas F, Guevara-Garcia A, Rosas G, et al. A new highly effective anticysticercosis vaccine expressed in transgenic papaya. Vaccine. 2007;25:4252-60.

15. Hernández M, Cervantes J, CabreraPonce JL, Herrera Estrella L, Rosas Salgado G, Villarreal ML, et al. Development of an oral anti-cysticercosis vaccine delivered in genetically modified embryogenic callus of papaya. Transgenic Plant J. 2011; en prensa. 